Journal of

Synchrotron

Radiation

ISSN 0909-0495

Received 18 November 2004

Accepted 10 March 2005

(C) 2005 International Union of Crystallography Printed in Great Britain - all rights reserved

\section{Overcoming protein denaturation caused by irradiation in a high-flux synchrotron radiation circular dichroism beamline}

\author{
Robert W. Janes* and Alison L. Cuff \\ School of Biological Sciences, Queen Mary, University of London, Mile End Road, London E1 4NS, \\ UK. E-mail: r.w.janes@qmul.ac.uk
}

It has been established that the new circular dichroism beamline CD12 has sufficiently high flux at low wavelengths to cause apparent irradiation problems with protein samples while their synchrotron radiation circular dichroism (SRCD) spectra are being collected. The cause of this effect has been extensively investigated and is reported in an accompanying paper [Wien et al. (2005). J. Synchrotron Rad. 12, 517-523.]. Experiments suggest that localized heating of the protein sample, leading to denaturation, is the probable cause. Methods to circumvent this problem by limiting the beam flux are reported. This was achieved using either an attenuation cell of water placed beam-side of the sample cell, or limiting the beam cross-sectional area hitting the sample. Such methods are shown to result in substantial reduction or apparent complete removal of this protein denaturation over the course of collecting three successive spectra. Elimination of this denaturation problem enables multiple SRCD scans for protein samples to be collected, which are vital both for good practice and for statistically valid results.

Keywords: circular dichroism; vacuum ultraviolet; protein denaturation; attenuation; protein structure.

\section{Introduction}

Circular dichroism (CD) spectroscopy is a technique that can be used to determine the secondary structure content of proteins; to observe and quantify structural changes that result from the binding of small molecules to proteins; and to follow the folding of proteins to their native conformations (Wallace, $2000 a, b)$. Conventional CD (cCD) measurements on laboratory-based machines have an accurate lower wavelength limit at best of $\sim 185 \mathrm{~nm}$, primarily because of limitations imposed by the drop in the flux from the source xenon arc lamps beyond this point. With this lower flux the signal from the sample becomes lost in the background noise levels. Much lower wavelength limits can be reached by switching to a synchrotron radiation light source where the flux is maintained at workable levels to wavelengths well below cCD machines (Sutherland et al., 1980). This extended range provides more data resulting in higher information content (Wallace \& Janes, 2001), thus more types of secondary structure can be identified and with greater resolution, more subtle changes can be observed in binding studies, and more can be determined about the nature of protein folding. Particularly for this last feature, this is due to the pronounced differences in the spectral characteristics displayed by the diverse types of secondary structure in the extended wavelength range, when compared with that seen in the range for $\mathrm{cCD}$ data (Wallace \& Janes, 2001). Synchrotron radiation CD (SRCD) therefore offers data with considerably greater potential for analyses over data collected from cCD machines.

CD12 is a new high-flux beamline on the SRS at Daresbury, UK, designed for collection of ultraviolet and vacuum-ultraviolet circular dichroism spectra over the extended range available from synchrotron radiation sources (Clarke \& Jones, 2004). The spectral characteristics of this beamline allow for SRCD data to be collected from $300 \mathrm{~nm}$ down to as low as $120 \mathrm{~nm}$ (Clarke \& Jones, 2004). During SRCD data collection on this beamline, we, and others (Wien et al., 2005; Clarke \& Jones, 2004), have observed notable changes in repeat spectra produced by protein samples that are clearly from some kind of irradiation effect. The suggestion from Clarke \& Jones (2004) is that this is a degradation process and may reflect the formation of radicals from the water solute in the sample cell, which in turn degrades the protein through free-radical damage. However, this irradiation effect has now been thoroughly investigated and the probable causes identified in an accompanying paper to this article (Wien et al., 2005). Our work has also shed light on the causes of the irradiation effect where we believe this to be denaturation of the protein. However, we primarily report on methods of circumnavigating this problem to allow SRCD data to be collected on CD12 
without any unexpected loss of the CD signal throughout the collection procedure.

\section{Materials and methodology}

The lysozyme and human serum albumin (HSA) used were purchased from Sigma Aldrich (UK). SRCD spectra were collected using different set-ups both to examine some of the properties of, and to try to prevent the effects of, irradiation on the protein sample. A stock solution of lysozyme was prepared at a concentration of approximately $20 \mathrm{mg} \mathrm{ml}^{-1}$, unless otherwise indicated. HSA at approximately $20 \mathrm{mg} \mathrm{ml}^{-1}$, the irradiation sensitivity of which is described in the accompanying paper (Wien et al., 2005), was used to show the significant improvement in spectral data collection that resulted when using attenuation. The protein samples were dialysed against water using Slide-A-Lyzer MINI dialysis units (Pierce, USA) and degassed under vacuum prior to data collection, as were the baseline solutions where used. Protein samples were mounted in a Hellma Quartz Suprasil demountable circular cell of pathlength $0.014 \mathrm{~mm}$. A second cell of pathlength $0.015 \mathrm{~mm}$ was also used, as described in subsequent sections [the pathlengths for each having been measured by interferometery techniques (Miles et al., 2005)]. All data were collected with the temperature for the sample set at $277 \mathrm{~K}$. All spectra were processed using CDTool (Lees et al., 2004).

\subsection{Overcoming irradiation effects with a water attenuator cell}

For all the spectra collected as reported in this section, a step size of $1 \mathrm{~nm}$ and a dwell time of $1 \mathrm{~s}$ were used. Three repeat scans were recorded on a lysozyme sample from stock using the $0.014 \mathrm{~mm}$ cell (Fig. 1a) over a wavelength range 300$170 \mathrm{~nm}$. All spectra were zeroed over the range $263-267 \mathrm{~nm}$. Using a fresh protein sample from the stock solution, an initial spectrum was recorded over the wavelength range 300$185 \mathrm{~nm}$; then the wavelength was set to $170 \mathrm{~nm}$ and the sample left exposed to the SRCD beam for $30 \mathrm{~min}$. This exposure

(a)

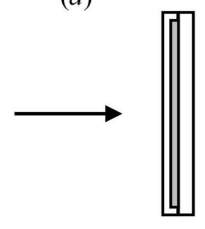

Figure 1

Arrangements of the cells for the experiments conducted. The arrow pointing to the right indicates the direction from the beam source. $(a)$ Initial set-up for the sample cell, directly in the SRCD beam. (b) Set-up for the water-filled beam-attenuated procedure. A water-filled cell is placed in the path of the beam prior to the sample. (c) Control set-up. Here the two glass cover plates are placed prior to the sample well plates which are now placed together creating one container, approximately double the size, as explained in the accompanying text. duration was equivalent to approximately five times that of a standard 300-185 nm scan. Following this, a further spectrum was recorded, repeating the protocol of the initial spectrum. The set-up for the attenuation procedure involved using a second cell (Fig. 1b). A water-filled cell, of pathlength $0.015 \mathrm{~mm}$, was placed into the sample holder, beam-side of the sample cell. Again an initial spectrum was recorded using a fresh protein sample from the stock. Here, however, the time scan at wavelength $170 \mathrm{~nm}$ was of $60 \mathrm{~min}$ duration, to counterbalance the presence of the attenuator cell. A further wavelength scan was then recorded. To test the validity of this attenuation procedure, and to clarify features of the irradiation effect, a control experiment was undertaken (Fig. 1c). Here, the two cell cover plates were placed prior to the sample holders. The protein sample was diluted to half its concentration $\left(\sim 10 \mathrm{mg} \mathrm{ml}^{-1}\right)$, then added to the $0.014 \mathrm{~mm}$ cell with sufficient volume that the $0.015 \mathrm{~mm}$ cell could be placed onto it (thereby creating almost exactly double the volume of sample in the beam), as shown in the Fig. 1(c). Spectra were then recorded in an identical fashion to that described for the water-cell attenuation experiment, the duration of the exposure again being $60 \mathrm{~min}$ because of the pathlength now being doubled. To test this attenuation procedure under more severe conditions, three spectra were collected with a step size of $0.5 \mathrm{~nm}$ over the wavelength range $300-170 \mathrm{~nm}$ using a $0.02 \mathrm{~mm}$-pathlength water-filled attenuator cell placed beamside of the $0.014 \mathrm{~mm}$ sample cell.

\subsection{Overcoming irradiation effects by limiting the cross- sectional area of the SRCD beam}

Four spectra of lysozyme were collected over the wavelength range $300-170 \mathrm{~nm}$ and with a step size of $0.5 \mathrm{~nm}$. The cross-sectional area of the incident SRCD beam hitting the sample was then reduced by closing the vertical slits on the beamline. When viewed from the source, the left- and righthand slits adjacent to the sample holder (controlled by the lower and upper dials, respectively, also adjacent to the sample holder) were set to 22.90 and 5.10 on their respective vernier scales. The detector current sensitivity, usually set at a value of 4.0, was reduced to 3.0 to compensate for the reduction in flux. Four spectra using a fresh protein sample from stock were then collected over the same wavelength range and with the same step size, for direct comparisons.

\subsection{Measuring HSA protein under limited cross-sectional area}

Four spectra of HSA were measured with a step size of $0.5 \mathrm{~nm}$ and dwell time of $1 \mathrm{~s}$, with the left- and right-hand slits reduced still further to vernier scale values of 23.25 and 4.75 , respectively. This was due to the substantial sensitivity to irradiation that had been observed for HSA by us (data not shown) and others (Wien et al., 2005). 


\section{Results and discussion}

Fig. 2 shows three sequential spectra of lysozyme collected on CD12 using a $1 \mathrm{~nm}$ step size and $1 \mathrm{~s}$ dwell time. When using a 'standard' set-up for collecting the SRCD data (Fig. 1a) there are substantial differences in these repeat spectra. These differences themselves are reproducible (data not shown), and indicate that the protein is undergoing some sort of alteration from the native state during the data collection as a result of being exposed to the SRCD beam. That a central cause of this irradiation effect is the high flux associated with CD12 is attested by the fact that this phenomenon was not seen in SRCD spectra of proteins collected on the lower-flux station 3.1, the now-decommissioned predecessor to CD12 (Orry et al., 2001). Nor is it seen on station UV1 on the ASTRID ring at Aarhus, Denmark (data not shown). There is a loss of magnitude in the signal between the first and second spectrum in the series, and between the second and third. This problem with the high flux means that in the set-up of the beamline used currently it has generally not been possible to collect reproducible spectra from a single sample. The most likely explanation for this is that some of the protein sample exposed

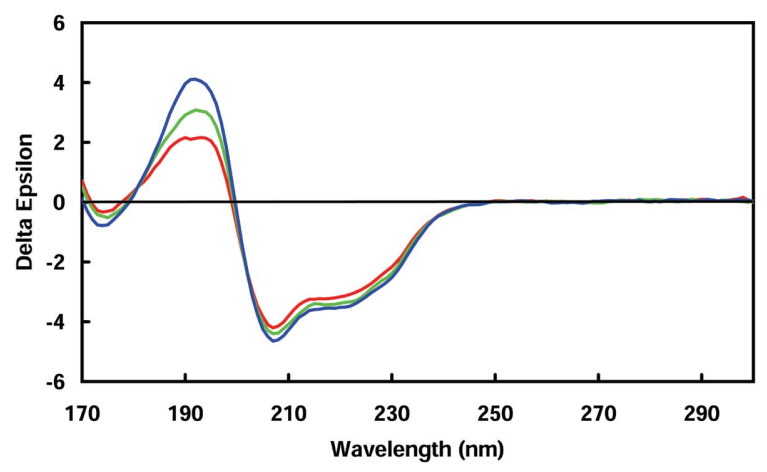

Figure 2

All spectra shown in this and subsequent figures were recorded over the wavelength range $300-170 \mathrm{~nm}$ unless otherwise stated. Three repeat spectra of lysozyme recorded under the initial set-up conditions (as in Fig. $1 a$ and described in §2.1). This clearly shows the effects of irradiation on the SRCD signal over the repeats from the first (blue) to the second (green) to the third (red).

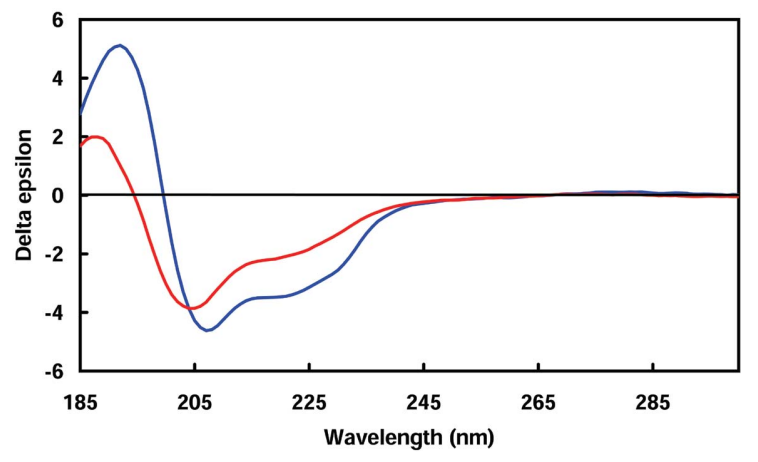

Figure 3

Spectra of lysozyme taken prior to exposure at $170 \mathrm{~nm}$ for $30 \mathrm{~min}$ in the SRCD beam under the initial set-up conditions (blue), and after exposure (red) (using the set-up as in Fig. $1 a$ and described in \$2.1). The recording range for these spectra was $300-185 \mathrm{~nm}$. to the SRCD beam is being affected by localized heating, but this is fully discussed in the accompanying paper (Wien et al., 2005).

Prior experiments had established that the irradiation problem was most severe after exposure to the flux from CD12 at wavelengths from $\sim 185 \mathrm{~nm}$ and lower (data not shown, and Wien et al., 2005; Clarke \& Jones, 2004). Fig. 3 shows spectra recorded before and after $30 \mathrm{~min}$ of exposure to the SRCD beam at $170 \mathrm{~nm}$ using the set-up shown in Fig. 1(b). Being approximately five times the duration taken to complete a single scan run, the 30 min exposure was significantly beyond the time necessary for a 'standard' three-scan experiment. Not only are there differences in the signal magnitude between the two spectra, but there are also peak shape and position differences as well. Fig. 4 shows the difference spectrum of the data in Fig. 3. This was created by scaling the CD spectral data of the 'after exposure' spectrum to be identical to the 'before exposure' spectrum at a wavelength of $222 \mathrm{~nm}$ (where no signal is seen for unordered structure), then subtracting the 'before exposure' data. This clearly demonstrates that being exposed to the high flux at $170 \mathrm{~nm}$ for $30 \mathrm{~min}$ results in the sample being denatured as shown by the spectrum in Fig. 4, characteristic of unordered protein.

By lowering the flux on the sample through attenuation, it was in turn expected that the irradiation effect would also be reduced, as the sample would not be so subjected to the localized heating during the data collection. Owing to the attenuation cell placed before the sample cell, both of approximately the same pathlength, the duration of exposure to the beam at $170 \mathrm{~nm}$ was doubled to provide a comparable total flux to that where no attenuation was used. Fig. 5 shows the results of this attenuation experiment. As can be seen, the difference in spectra before and after exposure to the beam at $170 \mathrm{~nm}$ is substantially smaller than that without attenuation. Attenuation of the beam in this manner therefore does appear to have significantly reduced the irradiation effect caused by the SRCD beam. It seems that the attenuation cell appears to have acted as a heat sink, absorbing the energy normally gained by the sample imparted to it by the high-flux beam. As the sample cell now receives lower flux, there is a significant

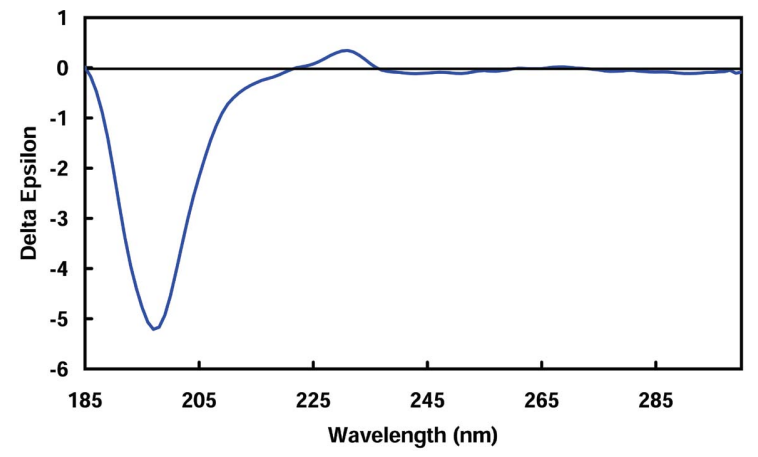

Figure 4

Difference spectrum of the spectra shown in Fig. 3, with the 'after exposure' spectrum first scaled to be identical to the 'before exposure' value at $222 \mathrm{~nm}$, then the 'before exposure' subtracted from it. 
reduction in the denaturation of the protein that results from the localized heating.

The spectra from the control experiment, conducted to ascertain whether the attenuation observed using the water cell was only because of an overall change in the pathlength and was thus nothing to do with heating, are given in Fig. 6, the set-up being shown in Fig. 1(c). Once again, exposure to the beam at $170 \mathrm{~nm}$ was doubled in time as the pathlength was also now double with the sample and attenuator cells being placed together. It is clear that there is no attenuation, and that increasing the pathlength alone has not overcome the irradiation effect. Indeed, the spectra shown in Fig. 6 are very similar to those in Fig. 3, where no attenuation was present, and are comparable with those observed from thermal denaturation experiments on lysozyme (Takesada et al., 1973). In addition, Fig. 4 agrees with this observation.

Fig. 7 shows three spectra of lysozyme now with a step size of $0.5 \mathrm{~nm}$ and using a $0.02 \mathrm{~mm}$-pathlength water-filled attenuator cell beam-side of the sample, since the reduced step size led to longer data-collection times and an increased exposure to the beam. There is substantial improvement in the comparability of the spectral shapes of the three spectra compared with those of Fig. 2.

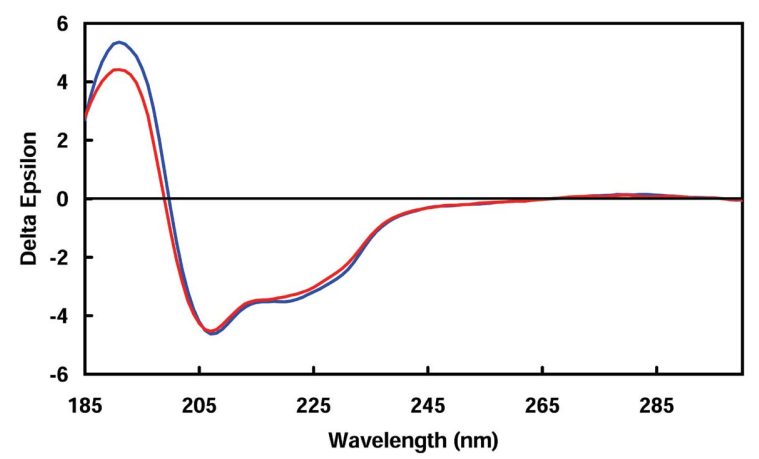

Figure 5

Spectra of lysozyme taken prior to exposure at $170 \mathrm{~nm}$ for $60 \mathrm{~min}$ in the SRCD beam under the attenuation conditions (blue) and after exposure (red) (using the set-up as in Fig. $1 b$ and described in \$2.1). The recording range for these spectra was $300-185 \mathrm{~nm}$.

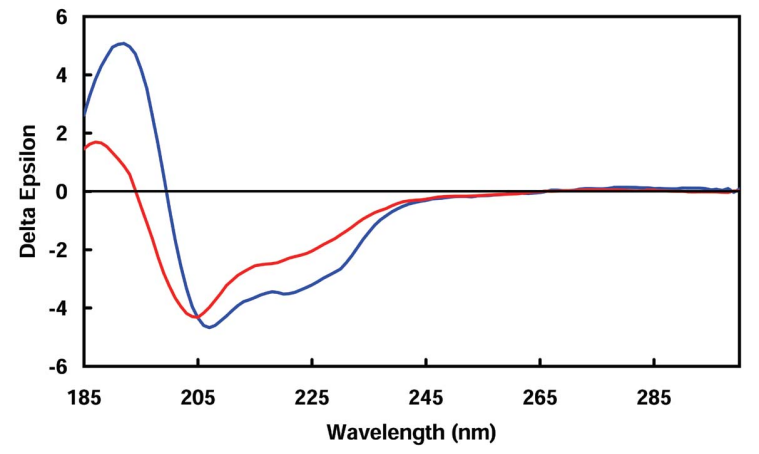

Figure 6

Spectra of lysozyme taken prior to exposure at $170 \mathrm{~nm}$ for $60 \mathrm{~min}$ in the SRCD beam under the control conditions (blue) and after exposure (red) (using the set-up as in Fig. $1 c$ and described in $\$ 2.1$ ). The recording range for these spectra was $300-185 \mathrm{~nm}$.
The water-cell attenuation approach greatly reduces the irradiation effect, even under the more severe conditions of data collection using a $0.5 \mathrm{~nm}$ scan step. However, there is still a slight loss of signal over the period of time and wavelength range of such a scanning protocol. Additionally, the water in the attenuator cell increases the absorption in the lower wavelength range to the detriment of measuring an accurate $\mathrm{CD}$ signal in this region, restricting the data collection as a result. Other liquids [ethylene glycol/water $(30 / 70 v / v)$ and fomblin oil (perfluoropolyether)] were examined for their suitability to act as the attenuator, but none have been identified so far to be any better in absorption properties at the lower wavelengths than the water itself (data not shown).

As collecting data in the lower wavelength range represents the major advantage of SRCD over cCD, other methods of attenuation to reduce the beam flux hitting the sample cell were explored. One such method used was to close the 'premonochromator' baffles on the beamline, the aim being to reduce the flux of the beam hitting the monochromator and thus reduce the overall flux reaching the sample. This approach, however, did not prove to be of use. Substantial reductions to one quarter of the initial voltages recorded on the detector (with no sample present, and used purely as a guide to the flux from the beamline) did not lessen the differences between spectra when a sample was irradiated (data not shown). A second approach was to close the vertical slits on the beamline adjacent to the sample chamber, hence limiting the cross-sectional area of the beam hitting the sample. Fig. 8 shows four spectra of the lysozyme stock protein recorded with a $0.5 \mathrm{~nm}$ step size, without attenuation and thus with the irradiation problem. With a duration of about $7 \mathrm{~min}$ for a scan, this equates to a radiation dose of around $4.4 \mathrm{~kJ} \mathrm{~m}^{-2}$ (Clarke \& Jones, 2004). The cross-sectional area of the full beam hitting the sample when measured photographically without the slits in place was found to be $\sim 5 \mathrm{~mm}$ $x \sim 12 \mathrm{~mm}$. The slits were then closed as described in $\$ 2.2$, the cross-sectional area of the beam being reduced to $\sim 5 \mathrm{~mm} \times$ $\sim 9 \mathrm{~mm}$, and four spectra were again recorded. The resultant spectra in Fig. 9 show that the differences between them are now within the overall noise of the spectra themselves. In this

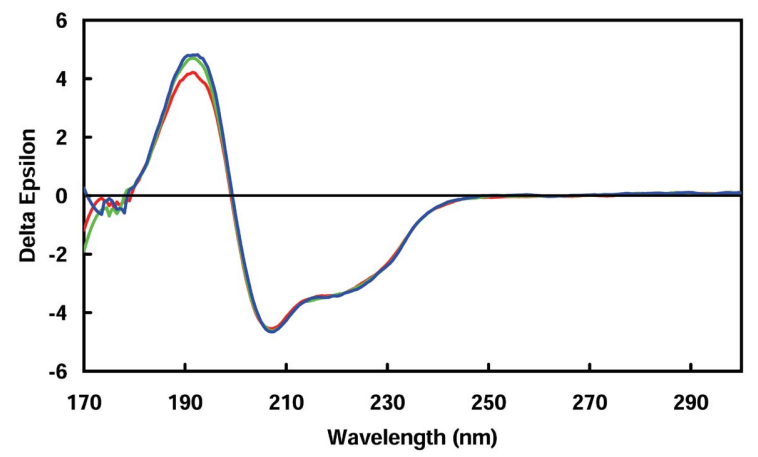

Figure 7

Three repeat spectra of lysozyme recorded with an attenuator in place and with a step size of $0.5 \mathrm{~nm}$ (blue, green and red being the first, second and third, respectively) (using the set-up as in Fig. $1 c$ and described in $\S 2.1)$. 


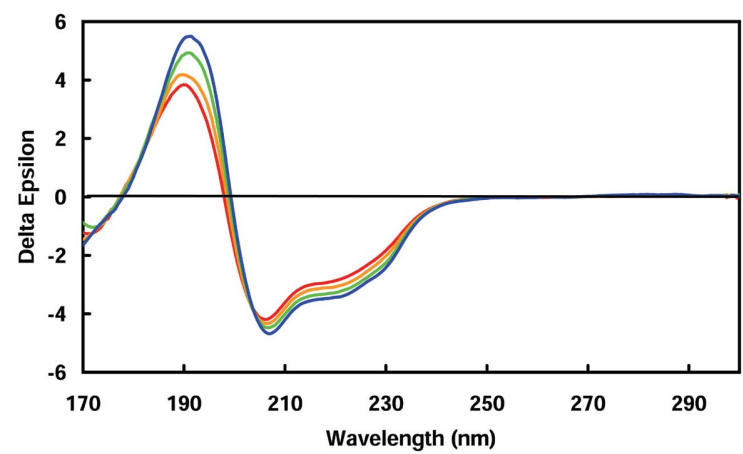

Figure 8

Four repeat spectra (blue, green, orange and red, respectively) of lysozyme unattenuated with a step size of $0.5 \mathrm{~nm}$. The trend observed in these spectra is similar to that seen in the spectra in Fig. 2 (using the setup as in Fig. $1 a$ and described in \$2.2).

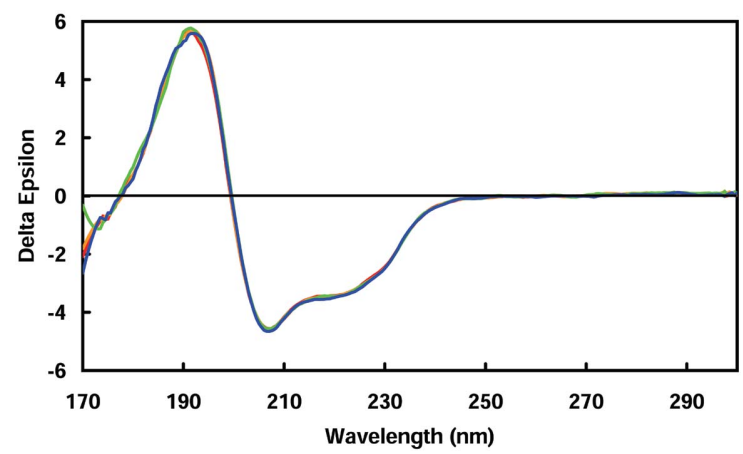

Figure 9

Four repeat spectra (blue, green, orange and red, respectively) of lysozyme with the beam slits closed to 5.10 and 22.90 on the vernier scales, and a step size of $0.5 \mathrm{~nm}$ (using the set-up as in Fig. $1 a$ and described in $\S 2.2)$.

slit configuration, three repeat spectra with a $0.5 \mathrm{~nm}$ step size can be recorded under good practice conditions without apparent denaturation of the protein sample. In order to test this set-up to the extreme, HSA was used. HSA has currently been found to be the protein most susceptible to the denaturation processes observed on CD12. With the slits closed as for the lysozyme spectra shown in Fig. 9, recording of HSA repeat spectra still gave rise to some denaturation, albeit to a very limited extent (data not shown). The slit width was thus reduced again to create a beam of $\sim 5 \mathrm{~mm} \times \sim 8 \mathrm{~mm}$, and the four spectra for HSA in Fig. 10 are the results from this position of the slits. For these four repeats there is no measurable denaturation, the differences between them remaining in the noise level. The signal-to-noise ratio for the spectra recorded using the non-attenuated, water-cell-attenuated and slit-attenuated procedures remained comparable in each case when examined over the wavelength range used for zeroing the spectra $(263-267 \mathrm{~nm})$.

Decreasing the slit width in this way from fully open to $\sim 5 \mathrm{~mm} \times \sim 8 \mathrm{~mm}$ represents a reduction from $\sim 60 \mathrm{~mm}^{2}$ to $\sim 40 \mathrm{~mm}^{2}$, approximately a decrease of one third in the crosssectional area of the source beam on the sample. The possibility exists that these attenuation experiments have in fact cut out stray light from hitting the sample, and that this is the root

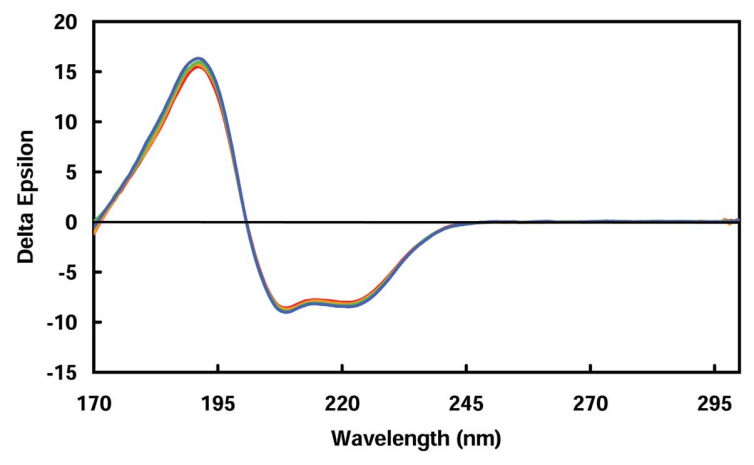

Figure 10

Four repeat spectra (blue, green, orange and red, respectively) of HSA with the beam slits closed to 4.75 and 23.25 on the vernier scales, and a step size of $0.5 \mathrm{~nm}$ (using the set-up as in Fig. $1 a$ and described in $\$ 2.3$ ).

cause of why spectra are subsequently reproducible. This cannot be excluded given the experiments conducted here; however, this is unlikely to be the case as numerous experiments have been conducted on this beamline which have concluded that any stray light is minimal to non-existent (D. Clarke, personal communication). In fact, closing the slits has probably introduced many beneficial effects for data collection. Less protein sample is being irradiated, and therefore there is less opportunity for the protein to be denatured. Additionally, and of significance, there is a greater area not being irradiated into which protein sample can diffuse away from the irradiation area. This would be a localized diffusion event induced by the localized heating in the sample by the high-flux intensity of the synchrotron beam, and not an overall bulk effect. Sample that is exposed to the beam therefore has a greater chance of diffusing out, and even if 'slightly denatured' it has the opportunity to refold in the cooler conditions of the surrounding sample area. Likewise, 'fresh sample' from the surrounding pool has had an opportunity to diffuse in replenishing the sample being irradiated with fully folded protein. Diffusion is therefore probably providing the stabilization of conditions necessary to ensure that, over the period of time it takes to record repeat spectra (here under the more severe data-collection protocol of a $0.5 \mathrm{~nm}$ scan step and four repeats), there is no appreciable loss of signal through denaturation. Therefore, collecting three repeat spectra under these conditions, as a good practice protocol, will not compromise the state of a protein sample to any significantly measurable extent. Additionally, this set-up now enables productive protein-melting studies to be undertaken on CD12 (F. Wien \& A. Miles, personal communications).

\section{Conclusions}

With CD12 at Daresbury there exists a state-of-the-art SRCD beamline capable of recording data to wavelengths substantially lower than for conventional laboratory-based $\mathrm{CD}$ machines. In the set-up of the beamline that has been running to date there has been a problem associated with recording repeat scans causing apparent denaturation of protein samples throughout the course of the experiment. Clarke \& Jones 
(2004) suggested this to be a degradation process caused by radical formation, which from the accompanying paper seems not to be the primary cause. They also advise for most measurements the high flux of the CD12 beamline allows for the collection of good signal-to-noise data from only a single rapid scan which would therefore avoid the degradation problem. Whilst substantially reducing this problem, this is not a solution as no statistical analyses can be conducted on a single scan, and fresh, possibly precious, samples would have to be used to carry out 'repeat' scans to provide any validation for the CD spectra recorded. The work reported here shows that the denaturation problem can readily be overcome by reducing the cross-sectional area of the source beam hitting the sample. Our results show that four spectra of HSA, a protein highly sensitive to this denaturation, can be recorded with differences between the spectra being within the noise. With the slits closed, limiting the cross-sectional area of the source beam hitting the sample, this new set-up for the CD12 beamline enables a typical good practice protocol of collecting three repeat spectra to be adopted, thus ensuring that statistically valid data can be collected.

This work was supported by a grant (B19312) from the BBSRC to RWJ, and grants from the CCLRC to RWJ for beam time at the SRS. We thank Farah O'Boyle for her help in support, and Professor B. A. Wallace, and her group, Dr Frank Wien, Jonathan Lees and Andrew Miles at Birkbeck College, London, and Professor John Sutherland, East Carolina University and Brookhaven National Laboratory, all for their very helpful discussions and suggestions.

\section{References}

Clarke, D. T. \& Jones, G. (2004). J. Synchrotron Rad. 11, 142-149.

Lees, J. G., Smith, B. R., Wien, F., Miles, A. J. \& Wallace, B. A. (2004). Anal. Biochem. 332, 285-289.

Miles, A. J., Wien, F., Lees, J. G. \& Wallace, B. A. (2005). Spectroscopy, 19, 43-51.

Orry, A. J. W., Janes, R. W., Sarra, R., Hanlon, M. R. \& Wallace, B. A. (2001). J. Synchrotron Rad. 8, 1027-1029.

Sutherland, J. C., Desmond, E. J. \& Takacs, P. Z. (1980). Nucl. Instrum. Methods, 172, 195-199.

Takesada, H., Nakamishi, M. \& Tsuboi, M. (1973). J. Mol. Biol. 77, 605-614.

Wallace, B. A. (2000a). Nature Struct. Biol. 7, 708-709.

Wallace, B. A. (2000b). J. Synchrotron Rad. 7, 289-295.

Wallace, B. A. \& Janes, R. W. (2001). Curr. Opin. Chem. Biol. 5, 567571.

Wien, F., Miles, A. J., Lees, J. G., Hoffmann, S. V. \& Wallace, B. A. (2005). J. Synchrotron Rad. 12, 517-523. 